\title{
Regularity for minimizers of functionals with nonstandard growth by A-harmonic approximation
}

\author{
Jens HABERMANN \\ Department of Mathematics \\ Friedrich-Alexander University \\ Bismarckstr. 1 1/2 \\ 91054 Erlangen, Germany \\ e-mail: habermann@mi.uni-erlangen.de \\ Anna ZATORSKA-GOLDSTEIN \\ Institute of Applied Mathematics and Mechanics \\ University of Warsaw \\ Banacha 2 \\ 00-913 Warsaw, Poland \\ e-mail: azator@mimuw.edu.pl
}

\begin{abstract}
We prove partial regularity for minimizers of quasiconvex functionals of the type $\int_{\Omega} f(x, D u) d x$ with $p(x)$ growth with respect to the second variable. The proof is direct and it uses a method of $\mathcal{A}$-harmonic approximation.

2000 Mathematics Subject Classification: 49N60, 35J50.

Key words: Quasiconvex integral functionals, nonstandard growth, $p(x)$ growth, partial regularity, $A$-harmonic approximation, Hölder continuity.
\end{abstract}

\section{Introduction}

In this paper we study the regularity properties of local minimizers of a variational functional

$$
\mathcal{F}[u]=\int_{\Omega} f(x, D u) d x,
$$

A.Z.-G. is partially supported by MEiN grant no 1PO3A 00529 and by Alexander von Humboldt Foundation 
where $u: \Omega \rightarrow \mathbb{R}^{N}, \Omega$ is a bounded domain in $\mathbb{R}^{n}$ and the integrand $f: \Omega \times$ $\operatorname{Hom}\left(\mathbb{R}^{n} ; \mathbb{R}^{N}\right) \rightarrow \mathbb{R}$ satisfies a growth condition of the type

$$
f(x, A) \approx\left(1+|A|^{2}\right)^{p(x) / 2},
$$

for $p: \Omega \rightarrow(1, \infty)$ being a Hölder continuous function. For the precise statement of the conditions see Section 2 .

Definition 1 A function $u \in W_{l o c}^{1,1}\left(\Omega ; \mathbb{R}^{n}\right)$ is called a local minimizer of the functional $\mathcal{F}$ if $|D u|^{p(x)} \in L_{l o c}^{1}(\Omega)$ and

$$
\mathcal{F}[u] \leq \mathcal{F}[u+\varphi]
$$

for all $\varphi \in W_{0}^{1,1}\left(\Omega ; \mathbb{R}^{N}\right)$ with compact support in $\Omega$.

The main statement is the following

Theorem 1 Let $u \in W_{l o c}^{1,1}\left(\Omega ; \mathbb{R}^{N}\right)$ be a local minimizer of the functional $\mathcal{F}$ fulfilling the assumptions $\mathbf{A 1}$ - $\mathbf{A} 3$ (see Section 2). Let $\gamma_{2}$ be an upper bound for the exponent $p$ and assume that the modulus of continuity $\omega$ of $p$ satisfies the condition

$$
\omega(\rho) \leq L \rho^{\alpha},
$$

for some $L>1, \alpha \in(0,1]$ and all $\rho<1$. Then there exists an open subset $\Omega_{0} \subset \Omega$ with $\mathcal{L}^{n}\left(\Omega \backslash \Omega_{0}\right)=0$ such that $D u \in C_{l o c}^{0, \beta}\left(\Omega_{0}\right)$ with $\beta=\min \left\{1, \frac{2}{\gamma_{2}}\right\} \frac{\alpha}{4}$.

The proof of the theorem (with different $\beta$ ) was first done by E. Acerbi and G. Mingione in 2001 [3]. The key step is to establish a certain decay estimate for the so called excess function $\Phi$, which is defined as

$$
\Phi \equiv \Phi\left(x_{0}, \rho, A\right) \equiv\left(f_{B_{\rho}\left(x_{0}\right)}\left|V_{p_{2}}(D u)-V_{p_{2}}(A)\right|^{2} d x\right)^{1 / 2}
$$

where $p_{2}$ denotes the maximum of the exponent function $p(x)$ in a neighbourhood of $x_{0}$ and with $V_{p}: \mathbb{R}^{k} \rightarrow \mathbb{R}^{k}$ given by

$$
V_{p}(\xi)=\left(1+|\xi|^{2}\right)^{(p-2) / 4} \xi
$$

The function $\Phi$ provides an integral measure of the oscillations of the gradient $D u$ in a ball $B_{\rho}$. The excess-decay estimate leads to Hölder continuity of $D u$ in $B_{\rho}$ via the integral characterization of Hölder continuous functions due to Campanato (see [6]). The excess-decay estimate was established by Acerbi and Mingione in an indirect way, using the blow-up technique.

We present here a more direct proof of the result. Applying the variational principle of Ekeland we obtain a comparison function, i.e. a function which is an 
almost minimizer of the functional with frozen $x$-coefficient and which is close to our local minimizer in an appropriate Sobolev norm. Having such a comparison function at hand, we are able to use the results for almost minimizers of variational functionals with constant $p$ growth. In particular, we obtain straightforward a Cacciopoli type inequality for local minimizers of the functional $\mathcal{F}$. Then, instead of blow-up arguments we use a method of $\mathcal{A}$-harmonic approximation to obtain an excess-decay estimate.

The method originates in a work of E. De Giorgi. It is based on the fact that one is able to obtain a good approximation of a function $w \in W^{1,2}\left(B ; \mathbb{R}^{N}\right)$, which is approximately $\mathcal{A}$-harmonic in a certain sense by an $\mathcal{A}$-harmonic function $h \in W^{1,2}\left(B ; \mathbb{R}^{N}\right)$, in both the $L^{2}$-topology and the weak topology in $W^{1,2}$. Here $h$ is called $\mathcal{A}$-harmonic on $B$ if there holds

$$
\int_{B} \mathcal{A}(D h, D \varphi) d x=0 \quad \text { for any } \varphi \in C_{0}^{1}\left(B ; \mathbb{R}^{N}\right),
$$

where $\mathcal{A}$ is a bilinear form on $\operatorname{Hom}\left(\mathbb{R}^{n} ; \mathbb{R}^{N}\right)$ which is (strongly) elliptic in the sense of Legendre-Hadamard, i.e. for all $\eta \in \mathbb{R}^{n}$ and $\xi \in \mathbb{R}^{N}$ there holds:

$$
\mathcal{A}(\eta \otimes \xi, \eta \otimes \xi) \geq \kappa|\eta|^{2}|\xi|^{2} .
$$

Since our results are of local nature, we can assume that our exponent function $p$ varies in some bounded interval $\left[\gamma_{1}, \gamma_{2}\right]$. However we have to be able to consider both cases $\gamma_{2} \geq 2$ and $1<\gamma_{2}<2$. For $p \geq 2$ it is straightforward to adapt the standard $\mathcal{A}$-harmonic approximation lemma by using the $L^{2}$-theory combined with a standard Sobolev inequality. For $1<p<2$ we do not have access to the $L^{2}$-theory for functions in $W^{1, p}$ but still it is possible to generalize the approximation lemma directly. This was done in [9].

Apart from the fact that the proof is very clear, the method provides better control of the constants. We have to admit that the proof of the $\mathcal{A}$-approximation lemma itself is done by contradiction. In direct way we show that a local minimizer is approximately $\mathcal{A}$-harmonic, where $\mathcal{A}=D^{2} f\left(x_{0},(D u)_{x_{0}, \rho}\right)$. The $\mathcal{A}$-harmonic approximation lemma guarantees the existence of a certain constant which is, admittedly not in an explicit form, determined by a property of constant coefficient elliptic systems, and we will later use the constant in the regularity proof. This constant, however, does not have an influence on the final Hölder exponent of $D u$. We should mention that by our method we end up with a final Hölder exponent

$$
\beta \equiv \min \left\{1, \frac{2}{\gamma_{2}}\right\} \frac{\alpha}{4}
$$

for the function $D u$, where $\alpha$ denotes the Hölder exponent of the exponent function $p$ and $\gamma_{2}$ is the global bound for $p$. This is, in fact, a slightly better result than stated in [3].

In order to obtain regularity results for local minimizers of the functional $\mathcal{F}$ we have to assume some continuity properties of the exponent $p$. Denoting by $\omega$ 
the modulus of continuity for the exponent function $p$ and at the same time for the $x$-dependence of the functional, the minimal condition for $\omega$ is that

$$
\limsup _{\rho \rightarrow 0} \omega(\rho) \log \left(\frac{1}{\rho}\right)<\infty .
$$

Dropping this assumption in general causes the loss of any type of regularity of minimizers (see [15]). By the result of Zhikov [14], (1.4) is sufficient to obtain higher integrability of the gradient of a minimizer. However, it is not sufficient to obtain further regularity. Acerbi and Mingione [3] proved $C^{0, \alpha}$ regularity for minimizers for every $\alpha<1$, provided the modulus of continuity satisfies an assumption

$$
\limsup _{\rho \rightarrow 0} \omega(\rho) \log \left(\frac{1}{\rho}\right)=0,
$$

which is in accordance with the theory of functionals with constant $p$-growth (in this case, $\omega$ denotes the modulus of continuity for the $x$-dependence of the functional). In order to prove $C^{1, \alpha}$ regularity of minimizers, in constant $p$ case (both for $p \in(1,2)$ and $p \geq 2$ ) assumption (1.5) is not sufficient. In fact one needs either that the modulus of continuity satisfies the so called Dini condition or that $p$ itself is Hölder continuous function, i.e.

$$
\omega(\rho) \approx \rho^{\alpha}
$$

This condition was assumed by Acerbi and Mingione in the original proof of the result of Hölder continuity of the gradient of a minimizer in $p(x)$ case.

\section{Setting}

We impose the following structure conditions for the functional $\mathcal{F}$ :

A1 (growth): the function $f(x, \cdot)$ is of the class $C^{2}$ and there exist constants $\mu \in(0,1], L \geq 1$ such that for all $x \in \Omega$ and $A \in \operatorname{Hom}\left(\mathbb{R}^{n} ; \mathbb{R}^{N}\right)$ we have

$$
L^{-1}|A|^{p(x)} \leq f(x, A) \leq L\left(\mu^{2}+|A|^{2}\right)^{p(x) / 2},
$$

where $p: \Omega \rightarrow(1, \infty)$ is a continuous function;

A2 (quasi-convexity): the function $f(x, \cdot)$ is (strictly) quasi-convex i.e.

$$
\begin{aligned}
\int_{B_{\rho}\left(x_{0}\right)}\left(f\left(x_{0}, A+D \varphi\right)-\right. & \left.f\left(x_{0}, A\right)\right) d x \\
& \geq \frac{1}{L} \int_{B_{\rho}\left(x_{0}\right)}\left(\mu^{2}+|A|^{2}+|D \varphi|^{2}\right)^{\frac{p\left(x_{0}\right)-2}{2}}|D \varphi|^{2} d x,
\end{aligned}
$$

for all $x_{0} \in \Omega, B_{\rho}\left(x_{0}\right) \Subset \Omega, A \in \operatorname{Hom}\left(\mathbb{R}^{n} ; \mathbb{R}^{N}\right)$ and $\varphi \in C_{0}^{\infty}\left(B_{\rho}\left(x_{0}\right) ; \mathbb{R}^{N}\right)$; 
A3 (continuity): the function $f$ satisfies the following continuity condition with respect to the first variable

$$
\begin{array}{r}
\left|f(x, A)-f\left(x_{0}, A\right)\right| \leq L \omega\left(\left|x-x_{0}\right|\right)\left[\left(\mu^{2}+|A|^{2}\right)^{p(x) / 2}+\left(\mu^{2}+|A|^{2}\right)^{p\left(x_{0}\right) / 2}\right] \\
\cdot\left[1+\left|\log \left(\mu^{2}+|A|^{2}\right)\right|\right],
\end{array}
$$

for all $x, x_{0} \in \Omega$ and $A \in \operatorname{Hom}\left(\mathbb{R}^{n}, \mathbb{R}^{N}\right)$, where $\omega:(0, \infty) \rightarrow(0, \infty)$ is a modulus of continuity for the function $p$, i.e. a non-decreasing, continuous function with $\lim _{R \rightarrow 0} \omega(R)=0$ and

$$
|p(x)-p(y)| \leq \omega(|x-y|) \quad \text { for all } x, y \in \Omega .
$$

Remark that $(2.8)$ is a natural condition for $f(x, A)=\left(1+|A|^{2}\right)^{p(x) / 2}$.

Since $f(x, \cdot)$ is quasi-convex and satisfies the growth condition (2.6) it is well known that there exists a constant $c=c(n, N, p(\cdot), L)$ such that the first derivatives of $f$ with respect to the second variable satisfy the growth condition

$$
\left|D f\left(x_{0}, A\right)\right| \leq c\left(1+|A|^{p\left(x_{0}\right)-1}\right) .
$$

We do not assume an explicit growth condition for the second derivatives of $f$. For our purposes it is sufficient that for any $M>0$ there exists a constant $K\left(M, x_{0}\right)>$ 0 such that for $A \in \operatorname{Hom}\left(\mathbb{R}^{n} ; \mathbb{R}^{N}\right)$

$$
\sup _{|A| \leq M}\left|D^{2} f\left(x_{0}, A\right)\right| \leq K\left(M, x_{0}\right) .
$$

Condition (2.6) implies also the existence of a modulus of continuity of $D^{2} f(x, \cdot)$ on compact subsets of $\operatorname{Hom}\left(\mathbb{R}^{n} ; \mathbb{R}^{N}\right)$ i.e. for any given constant $M>0$

$$
\left|D^{2} f\left(x_{0}, A\right)-D^{2} f\left(x_{0}, B\right)\right| \leq \nu_{M, x_{0}}(|A-B|),
$$

for any $A, B \in \operatorname{Hom}\left(\mathbb{R}^{n}, \mathbb{R}^{N}\right)$ with $|A|,|B| \leq M+1$.

Remark 1 Since our results are of local nature we will assume that there exist $1<\gamma_{1} \leq \gamma_{2}<\infty$ such that

$$
\gamma_{1} \leq p(x) \leq \gamma_{2} \quad \text { for all } x \in \Omega .
$$

It follows that $K\left(M, x_{0}\right)$ in $(2.10)$ may be chosen independently of $x_{0}$. We will therefore omit its dependence on $x_{0}$ and write $K(M)$.

Some notation: Within the whole paper we will write $B\left(x_{0}, \rho\right)$ for the open ball with centre $x_{0} \in \mathbb{R}^{n}$ and radius $\rho$. Furthermore we write

$$
(u)_{x_{0}, \rho} \equiv f_{B\left(x_{0}, \rho\right)} u d x \equiv \frac{1}{\left|B\left(x_{0}, \rho\right)\right|} \int_{B\left(x_{0}, \rho\right)} u d x
$$


for the mean value of the function $u$ on the ball $B\left(x_{0}, \rho\right)$. From time to time we just write $B_{\rho}\left(x_{0}\right)$, or if the center is clear from the context, $B_{\rho}$ instead of $B\left(x_{0}, \rho\right)$. The same we do with the notation for the mean value, i.e. we just write $(u)_{\rho}$ instead of $(u)_{x_{0}, \rho}$. Concerning the constants appearing in the proofs we remark that they may change from line to line. If a constant will be important for the proceeding of the proofs, we will indicate this in an obvious way. From time to time for clearness we will not show the dependencies of the constants within the estimates, but at the end of them.

\section{Basic tools}

\subsection{Higher integrability}

We start with a higher integrability result due to Zhikov, which in the form of the following statement appears in [2].

Lemma 2 Let $u \in W_{l o c}^{1,1}\left(\Omega, \mathbb{R}^{N}\right)$ with $|D u|^{p(\cdot)} \in L_{l o c}^{1}$ be a local minimizer of the functional

$$
w \mapsto \int_{\Omega} f(x, D w(x)) d x,
$$

where $f$ satisfies the growth and ellipticity conditions (2.6), (2.7) and assumption (1.4) on the modulus of continuity $\omega$ holds. Furthermore assume that

$$
\int_{\Omega}|D u|^{p(x)} d x \leq M<\infty .
$$

Then there exist an exponent $\delta=\delta\left(n, \gamma_{1}, \gamma_{2}, L, M\right)>0$, a constant $c=$ $c\left(n, \gamma_{1}, \gamma_{2}, L, M\right)$ and a radius $R_{0}=R_{0}\left(n, \gamma_{1}, \omega(\cdot)\right)$ such that for any radius $0<R \leq R_{0}$ there holds

$$
\left(f_{B_{R / 2}}|D u|^{p(x)(1+\delta)} d x\right)^{\frac{1}{1+\delta}} \leq c\left(f_{B_{R}}|D u|^{p(x)} d x+1\right) .
$$

The next lemma is an up-to-the-boundary result. The version we present here, with balls of the same size on both sides of the inequality was proved in [13] in higher order case. The original proof (with a ball of double radius on the right hand side) can be found in [1], [3], [8].

Lemma 3 (Higher integrability up to the boundary) Let $p>1$ and $B\left(x_{0}, \rho\right) \Subset \Omega$. Assume $g: \Omega \times \mathbb{R}^{n N} \rightarrow \mathbb{R}$ is continuous and for all $z \in \mathbb{R}^{n N}$ there holds

$$
L^{-1}|z|^{p} \leq g(x, z) \leq L\left(|z|^{p}+a(x)\right),
$$

with $L \geq 1,0<a \in L^{\gamma}\left(B_{\rho}\right), \gamma>1$. 
Let $h \in W^{1, q}\left(B_{\rho}\right)$ with $q>p$ and $v$ be a solution of the Dirichlet problem

$$
\min \left\{\int_{B_{\rho}} g(x, D w) d x, \quad w \in h+W_{0}^{1, p}\left(B_{\rho}\right)\right\} .
$$

Then there exists $\varepsilon=\varepsilon(p, L, m) \in(0, m)$ with $m=\min \left\{\gamma-1, \frac{q}{p}-1\right\}$ and $a$ constant $c \equiv c(p, L)$ such that

$$
\begin{aligned}
\left(f_{B_{\rho}}|D v|^{p(1+\varepsilon)} d x\right)^{\frac{1}{p(1+\varepsilon)}} \leq c\left[\left(f_{B_{\rho}}|D v|^{p} d x\right)^{\frac{1}{p}}\right. & +\left(f_{B_{\rho}}|D h|^{p(1+m)} d x\right)^{\frac{1}{p(1+m)}} \\
& \left.+\left(f_{B_{\rho}} a^{1+m} d x\right)^{\frac{1}{p(1+m)}}\right] .
\end{aligned}
$$

Remark 2 In the case of $p \in\left[\gamma_{1}, \gamma_{2}\right]$ with $1<\gamma_{1} \leq \gamma_{2}<+\infty$, we can choose $\varepsilon$ and $c$ independent of $p$ itself, replacing the dependence on $p$ by a dependence on $\gamma_{1}$ and $\gamma_{2}$.

\subsection{Ekeland variational principle}

In order to obtain a comparison function, i.e. an almost minimizer of the functional with frozen coefficients we apply a well known variational principle of Ekeland (see [11]).

Lemma 4 Let $(X, d)$ be a complete metric space and $\mathcal{G}: X \rightarrow(-\infty,+\infty]$ a lower semicontinuous functional such that $\inf _{X} \mathcal{G}$ is finite. Given $\varepsilon>0$ let $u \in X$ be such that $\mathcal{G}(u) \leq \inf _{X} \mathcal{G}+\varepsilon$. Then there exists $w \in X$ such that

$$
\begin{aligned}
d(w, u) & \leq 1 \\
\mathcal{G}(w) & \leq \mathcal{G}(u), \\
\mathcal{G}(w) & \leq \mathcal{G}(v)+\varepsilon d(v, w), \quad \text { for any } v \in X .
\end{aligned}
$$

\subsection{Algebraic properties of the function $V_{p}$}

Let the function $V \equiv V_{p}: \mathbb{R}^{k} \rightarrow \mathbb{R}^{k}$ be defined by

$$
V_{p}(z)=\left(1+|z|^{2}\right)^{\frac{p-2}{4}} z .
$$

We recall algebraic properties of the function $V_{p}$ (for a proof of the properties see e.g. [8]).

Lemma 5 Let $p>1$ and let $V \equiv V_{p}: \mathbb{R}^{k} \rightarrow \mathbb{R}^{k}$ be as in (3.14). Then for any $z, \eta \in \mathbb{R}^{k}$ there holds 
i) $|V(t z)| \leq \max \left\{t, t^{p / 2}\right\}|V(z)|$, for any $t>0$;

ii) $|V(z+\eta)| \leq c(|V(z)|+|V(\eta)|)$;

iii)

$$
c^{-1}|z-\eta| \leq \frac{|V(z)-V(\eta)|}{\left(1+|z|^{2}+|\eta|^{2}\right)^{(p-2) / 4}} \leq c|z-\eta| ;
$$

Moreover for any $z \in \mathbb{R}^{k}$

iv)

$$
\begin{aligned}
\text { if } p \in(1,2): & \frac{1}{\sqrt{2}} \min \left\{|z|,|z|^{p / 2}\right\} \leq|V(z)| \leq \min \left\{|z|,|z|^{p / 2}\right\} ; \\
\text { if } p \geq 2: & \max \left\{|z|,|z|^{p / 2}\right\} \leq|V(z)| \leq \sqrt{2} \max \left\{|z|,|z|^{p / 2}\right\} ;
\end{aligned}
$$

v)

$$
\begin{aligned}
\text { if } p \in(1,2): & |V(z)-V(\eta)| \leq c|V(z-\eta)|, \quad \text { for any } \eta \in \mathbb{R}^{k} ; \\
\text { if } p \geq 2: & |V(z)-V(\eta)| \leq c(M)|V(z-\eta)|, \quad \text { for }|\eta| \leq M ;
\end{aligned}
$$

vi)

$$
\begin{aligned}
\text { if } p \in(1,2): & |V(z-\eta)| \leq c(M)|V(z)-V(\eta)|, \quad \text { for }|\eta| \leq M ; \\
\text { if } p \geq 2: & |V(z-\eta)| \leq c|V(z)-V(\eta)|, \quad \text { for } \text { any } \eta \in \mathbb{R}^{k} ;
\end{aligned}
$$

with $c(M), c \equiv c(k, p)>0$. If $1<\gamma_{1} \leq p \leq \gamma_{2}$ all the constants $c(k, p)$ may be replaced by a single constant $c \equiv c\left(k, \gamma_{1}, \gamma_{2}\right)$.

\section{4 $\mathcal{A}$-harmonic approximation and a priori estimates for $\mathcal{A}$-harmonic functions}

The key ingredient of the proof is the following $\mathcal{A}$-harmonic approximation lemma. The proof for the case $p \geq 2$ can be found in [10]. The case $1<p<2$ has been proved in [9].

Lemma 6 Let $p>1$ and $\kappa, K$ be positive constants. Then for any $\varepsilon>0$ there exists $\delta=\delta(n, N, \kappa, K, \varepsilon) \in(0,1]$ with the following property: for any bilinear form $\mathcal{A}$ on $\operatorname{Hom}\left(\mathbb{R}^{n} ; \mathbb{R}^{N}\right)$ which is elliptic in the sense of Legendre-Hadamard with ellipticity constant $\kappa$ and upper bound $K$ and for any $v \in W^{1, p}\left(B_{\rho}\left(x_{0}\right) ; \mathbb{R}^{N}\right)$ satisfying

$$
f_{B_{\rho}\left(x_{0}\right)}\left|V_{p}(D v)\right|^{2} d x \leq \gamma^{2} \leq 1 \quad \text { and }
$$




$$
f_{B_{\rho}\left(x_{0}\right)} \mathcal{A}(D v, D \varphi) d x \leq \gamma \delta \sup _{B_{\rho}\left(x_{0}\right)}|D \varphi| \quad \text { for all } \varphi \in C_{0}^{1}\left(B_{\rho}\left(x_{0}\right) ; \mathbb{R}^{N}\right),
$$

there exists an $\mathcal{A}$-harmonic function $h$ satisfying

$$
f_{B_{\rho}\left(x_{0}\right)}\left|V_{p}(D h)\right|^{2} d x \leq c \quad \text { and } \quad f_{B_{\rho}\left(x_{0}\right)}\left|V_{p}\left(\frac{v-\gamma h}{\rho}\right)\right|^{2} d x \leq c \gamma^{2} \varepsilon,
$$

with a constant $c=c\left(\gamma_{1}, \gamma_{2}\right)$.

In Section 5.1 we will use a priori estimates for solutions of linear elliptic systems of second order with constants coefficients (see e.g. [8] and [9]).

Lemma 7 Let $\mathcal{A}$ be a bilinear form on $\operatorname{Hom}\left(\mathbb{R}^{n} ; \mathbb{R}^{N}\right)$ which is elliptic in the sense of Legendre-Hadamard with ellipticity constant $\kappa$ and upper bound $K$ and assume that $h \in W^{1,1}\left(B_{\rho}\left(x_{0}\right) ; \mathbb{R}^{N}\right)$ is an $\mathcal{A}$-harmonic function, i.e.

$$
\int_{B_{\rho}\left(x_{0}\right)} \mathcal{A}(D h, D \varphi) d x=0,
$$

for any $\varphi \in C_{0}^{1}\left(B_{\rho}\left(x_{0}\right) ; \mathbb{R}^{N}\right)$. Then $h \in C^{\infty}\left(B_{\rho}\left(x_{0}\right) ; \mathbb{R}^{N}\right)$ and

$$
\rho \sup _{B_{\rho / 2}\left(x_{0}\right)}\left|D^{2} h\right|+\sup _{B_{\rho / 2}\left(x_{0}\right)}|D h| \leq c_{a} f_{B_{\rho}\left(x_{0}\right)}|D h| d x,
$$

where the constant $c_{a}$ depends only on $n, N, \kappa$ and $K$.

\section{Preliminary results}

As remarked before, since our results are of the local nature we will assume that there exist $1<\gamma_{1} \leq \gamma_{2}<\infty$ such that

$$
\gamma_{1} \leq p(x) \leq \gamma_{2} \quad \text { for all } x \in \Omega,
$$

and moreover

$$
\int_{\Omega}|D u|^{p(x)} d x<\infty .
$$

Let $\delta$ be the higher integrability exponent from Lemma 2 and let from now on the radius $R$ be so small that

$$
\omega(R) \leq \frac{\delta}{4} .
$$

Subsequently we will always assume that $\rho \leq R / 4$. Take a ball $B_{2 \rho}\left(x_{0}\right)$ and define

$$
p_{1}:=\inf \left\{p(x): x \in B_{2 \rho}\left(x_{0}\right)\right\}, \quad p_{2}:=\sup \left\{p(x): x \in B_{2 \rho}\left(x_{0}\right)\right\} .
$$


Let furthermore $x_{m} \in \overline{B_{2 \rho}\left(x_{0}\right)}$ be a point, where the function $p$ reaches the value $p_{2}$, i.e. $p_{2} \equiv p\left(x_{m}\right)$. Then by $p_{2}-p_{1} \leq \omega(R) \leq \delta / 4$ we get

$$
p_{2}(1+\delta / 4) \leq p_{1}(1+\delta) \leq p(x)(1+\delta) .
$$

\subsection{Comparison}

Proposition 1 Let $B_{2 \rho}\left(x_{0}\right) \Subset \Omega$ and assume that $\left(|D u|^{p_{2}}\right)_{x_{0}, 2 \rho} \leq \bar{M}<\infty$. Then there exist constants $C(\bar{M})=C\left(\bar{M}, \gamma_{1}, \gamma_{2}, L, \alpha\right)>0, c=c\left(\gamma_{1}, \gamma_{2}\right)>0$, and $a$ function $w \in u+W_{0}^{1, p_{2}}\left(B_{\rho}\left(x_{0}\right) ; \mathbb{R}^{N}\right)$ such that

$$
f_{B_{\rho}\left(x_{0}\right)}|D u-D w|^{p_{2}} d x \leq C(\bar{M}) \rho^{p_{2} \alpha / 2},
$$

and

$$
\begin{aligned}
& f_{B_{\rho}\left(x_{0}\right)} f\left(x_{m}, D w\right) d x \\
& \quad \leq f_{B_{\rho}\left(x_{0}\right)} f\left(x_{m}, D w+D \varphi\right) d x+c \rho^{\alpha / 2} f_{B_{\rho}\left(x_{0}\right)}\left(1+|D \varphi|^{p_{2}}\right) d x,
\end{aligned}
$$

for any $\varphi \in W_{0}^{1, p_{2}}\left(B_{\rho}\left(x_{0}\right) ; \mathbb{R}^{N}\right)$.

Proof. Consider the function $g(z):=f\left(x_{m}, z\right)$. Then $g$ satisfies the growth condition (2.6) with exponent $p_{2}=p\left(x_{m}\right)$. Let $v \in u+W_{0}^{1, p_{2}}\left(B_{\rho} ; \mathbb{R}^{N}\right)$ be the unique solution of the Dirichlet problem

$$
\min \left\{\int_{B_{\rho}} g(D w): w \in u+W_{0}^{1, p_{2}}\left(B_{\rho} ; \mathbb{R}^{N}\right)\right\} .
$$

$v$ exists as $f$ is quasiconvex. Lemma 3 with $p \equiv p_{2}, q \equiv p_{2}(1+\delta / 4)$ and $a(x) \equiv 1$ provides $\varepsilon \equiv \varepsilon\left(\gamma_{1}, \gamma_{2}, L\right)$ and $c \equiv c\left(\gamma_{1}, \gamma_{2}, L\right)$ with $0<\varepsilon<\delta / 4$ such that

$$
\begin{aligned}
& \left(f_{B_{\rho}\left(x_{0}\right)}|D v|^{p_{2}(1+\varepsilon)} d x\right)^{\frac{1}{p_{2}(1+\varepsilon)}} \\
& \quad \leq c\left(f_{B_{\rho}\left(x_{0}\right)}|D v|^{p_{2}} d x\right)^{1 / p_{2}}+\left(f_{B_{\rho}\left(x_{0}\right)}\left(|D u|^{p_{2}}+1\right)^{1+\delta / 4} d x\right)^{\frac{1}{p_{2}(1+\delta / 4)}} .
\end{aligned}
$$

Higher integrability for the function $u$ (Lemma 2) gives us

$$
f_{B_{\rho}}|D u|^{p_{2}(1+\delta / 4)} d x \leq C(\bar{M}) .
$$


For $v$ we get by the minimality and the growth condition

$$
f_{B_{\rho}}|D v|^{p_{2}} d x \leq L^{2} f_{B_{\rho}}\left(1+|D u|^{p_{2}}\right) d x
$$

so that together with the estimate before and the higher integrability for $u$ we have

$$
f_{B_{\rho}}|D v|^{p_{2}(1+\varepsilon)} d x \leq C(\bar{M})
$$

with $0<\varepsilon<\delta / 4$ and $C(\bar{M})$ also depending on $n, L, \gamma_{1}, \gamma_{2}$. We now estimate the difference

$$
\begin{aligned}
f_{B_{\rho}}(g(D u)-g(D v)) d x & =f_{B_{\rho}}\left(f\left(x_{m}, D u\right)-f\left(x_{m}, D v\right)\right) d x \\
= & f_{B_{\rho}}(f(x, D u)-f(x, D v)) d x+f_{B_{\rho}}(f(x, D v) \\
& \left.\quad-f\left(x_{m}, D v\right)\right) d x+f_{B_{\rho}}\left(f\left(x_{m}, D u\right)-f(x, D u)\right) d x \\
= & (I)+(I I)+(I I I),
\end{aligned}
$$

with the obvious labelling. By the minimality of $u$ we have $(I) \leq 0$. For $(I I I)$ we get by the continuity of $f$ in the first variable and the higher integrability of $u$

$$
|(I I I)| \leq c(\delta) \omega(4 \rho) f_{B_{\rho}}\left(|D u|^{p_{2}(1+\delta / 4)}+1\right) d x \leq C(\bar{M}) \omega(4 \rho) .
$$

By the same arguments for the function $v$ we have

$$
|(I I)| \leq c(\varepsilon) \omega(4 \rho) f_{B_{\rho}}\left(|D v|^{p_{2}(1+\varepsilon)}+1\right) d x \leq C(\bar{M}) \omega(4 \rho),
$$

so overall, using (1.1) we get

$$
f_{B_{\rho}}[g(D u)-g(D v)] d x \leq C \omega(4 \rho) \leq C(\bar{M}) \rho^{\alpha},
$$

with the constant $C(\bar{M})$ depending additionally on $n, L, \gamma_{1}, \gamma_{2}, \alpha$. Let $0<\mu<\alpha$, $X:=u+W_{0}^{1, p_{2}}\left(B_{\rho} ; \mathbb{R}^{N}\right)$ and

$$
d: X \times X \rightarrow[0, \infty), \quad d(z, w)=\frac{1}{C(\bar{M}) \rho^{\mu}}\left(f_{B_{\rho}}|D(z-w)|^{p_{2}} d x\right)^{1 / p_{2}}
$$


On the complete metric space $(X, d)$ we consider the functional

$$
\mathcal{G}: X \rightarrow \mathbb{R}, \mathcal{G}(z):=\int_{B_{\rho}} g(D z) d x
$$

which is clearly lower semicontinuous. By $\mathcal{G}(v)=\min _{X} \mathcal{G}$ and (4.18) we have

$$
\mathcal{G}(u) \leq \inf _{X} \mathcal{G}+C(\bar{M}) \rho^{\alpha} .
$$

Therefore the Ekeland variational principle (Lemma 4) provides a function $w \in$ $u+W_{0}^{1, p_{2}}\left(B_{\rho} ; \mathbb{R}^{N}\right)$ with the properties

$$
\begin{aligned}
f_{B_{\rho}}|D u-D w|^{p_{2}} d x & \leq C(\bar{M}) \rho^{\mu p_{2}} \quad \text { and } \\
f_{B_{\rho}} g(D w) d x & \leq f_{B_{\rho}} g(D w+D \varphi) d x+\rho^{\alpha-\mu}\left(f_{B_{\rho}}|D \varphi|^{p_{2}} d x\right)^{1 / p_{2}},
\end{aligned}
$$

for all $\varphi \in W_{0}^{1, p_{2}}\left(B_{\rho} ; \mathbb{R}^{N}\right)$. We estimate the second integral of the second inequality simply applying Bernoulli's inequality:

$$
\left(f_{B_{\rho}}|D \varphi|^{p_{2}} d x\right)^{1 / p_{2}} \leq\left(1+f_{B_{\rho}}|D \varphi|^{p_{2}} d x\right)^{1 / p_{2}} \leq c\left(\gamma_{1}, \gamma_{2}\right) f_{B_{\rho}}\left(1+|D \varphi|^{p_{2}}\right) d x
$$

Thus choosing $\mu \equiv \alpha / 2$ we obtain the assertion.

\subsection{Caccioppoli inequality}

Lemma 8 Let $\bar{M}, \hat{M}>0$. Assume that $u$ is a local minimizer of the functional $\mathcal{F}$ with $\left(|D u|^{p_{2}}\right)_{x_{0}, 2 \rho} \leq \bar{M}$ and $A \in \operatorname{Hom}\left(\mathbb{R}^{n} ; \mathbb{R}^{N}\right)$ with $|A| \leq \hat{M}$. There exist constants $\rho_{0}=\rho_{0}(\bar{M}, \hat{M}, \alpha)$ and $c_{c}=c_{c}(\hat{M}, \alpha)$ such that for every $\xi \in \mathbb{R}^{N}$ and every ball $B\left(x_{0}, \rho\right) \Subset \Omega$ with $\rho \leq \rho_{0}$ there holds:

$$
\begin{aligned}
& f_{B_{\rho / 2}\left(x_{0}\right)}\left|V_{p_{2}}(D u-A)\right|^{2} d x \\
& \leq c_{c}\left[f_{B_{\rho}\left(x_{0}\right)}\left|V_{p_{2}}\left(\frac{u-\xi-A\left(x-x_{0}\right)}{\rho}\right)\right|^{2} d x+\rho^{\alpha / 2}\right] .
\end{aligned}
$$

Proof. By Proposition 1 there exists an almost minimizer $w$ of the frozen functional $\tilde{\mathcal{F}}_{\Omega}[\cdot] \equiv \int_{B_{\rho}\left(x_{0}\right)} f\left(x_{m}, \cdot\right) d x$, such that

$$
f_{B_{\rho}\left(x_{0}\right)}|D u-D w|^{p_{2}} d x \leq C(\bar{M}) \rho^{p_{2} \alpha / 2},
$$


and $w$ satisfies (4.17), so that by Lemma 3 in [9] with $\omega(\rho)=\rho^{\alpha / 2}$ we have

$$
f_{B_{\rho / 2}\left(x_{0}\right)}\left|V_{p_{2}}(D w-A)\right|^{2} d x \leq c f_{B_{\rho}\left(x_{0}\right)}\left|V_{p_{2}}\left(\frac{w-\xi-A\left(x-x_{0}\right)}{\rho}\right)\right|^{2} d x+c \rho^{\alpha / 2},
$$

where $c=c(\hat{M})$. By Lemma $5(i i)$ and Hölder's inequality we have in the case $p_{2} \geq 2$

$$
\begin{aligned}
f_{B_{\rho / 2}} \mid V_{p_{2}} & \left.(D u-D w)\right|^{2} d x \\
& \leq f_{B_{\rho / 2}}|D u-D w|^{p_{2}} d x+\left(f_{B_{\rho / 2}}|D u-D w|^{p_{2}} d x\right)^{2 / p_{2}} \\
& \leq c\left(\rho^{p_{2} \alpha / 2}+\rho^{\alpha}\right)
\end{aligned}
$$

In the case $1<p_{2}<2$ we directly estimate

$$
f_{B_{\rho / 2}\left(x_{0}\right)}\left|V_{p_{2}}(D w-A)\right|^{2} d x \leq f_{B_{\rho / 2}\left(x_{0}\right)}|D u-D w|^{p_{2}} d x \leq c \rho^{p_{2} \alpha / 2}
$$

and thus in every case we have

$$
\begin{aligned}
& f_{B_{\rho / 2}}\left|V_{p_{2}}(D u-A)\right|^{2} d x \\
& \leq c\left(p_{2}\right)\left[f_{B_{\rho / 2}}\left|V_{p_{2}}(D u-D w)\right|^{2} d x+f_{B_{\rho / 2}}\left|V_{p_{2}}(D w-A)\right|^{2} d x\right] \\
& \leq c\left(p_{2}\right)\left[\rho^{p_{2} \alpha / 2}+\rho^{\alpha}+f_{B_{\rho / 2}}\left|V_{p_{2}}(D w-A)\right|^{2} d x\right] .
\end{aligned}
$$

On the other hand, again by the properties of the function $V_{p_{2}}$ we estimate

$$
\begin{aligned}
& f_{B_{\rho}}\left|V_{p_{2}}\left(\frac{w-\xi-A\left(x-x_{0}\right)}{\rho}\right)\right|^{2} d x \\
& \quad \leq c\left(p_{2}\right)\left[f_{B_{\rho}}\left|V_{p_{2}}\left(\frac{u-\xi-A\left(x-x_{0}\right)}{\rho}\right)\right|^{2} d x+f_{B_{\rho}}\left|V_{p_{2}}\left(\frac{w-u}{\rho}\right)\right|^{2} d x\right] .
\end{aligned}
$$

Since $u-w \in W_{0}^{1, p_{2}}\left(B_{\rho} ; \mathbb{R}^{N}\right)$, we apply Poincaré's inequality on the second term of the right hand side, finally obtaining (again using properties of the function $V_{p_{2}}$ )

$$
f_{B_{\rho}}\left|V_{p_{2}}\left(\frac{u-w}{\rho}\right)\right|^{2} d x \leq c f_{B_{\rho}}\left|V_{p_{2}}(D w-D u)\right|^{2} d x \leq c\left(\rho^{p_{2} \alpha / 2}+\rho^{\alpha}\right) .
$$


Hence from (4.20) and (4.21) we obtain

$$
\begin{aligned}
& f_{B_{\rho / 2}\left(x_{0}\right)} \mid V_{p_{2}}(D u-A)\left.\right|^{2} d x \\
& \leq c\left[\rho^{p_{2} \alpha / 2}+f_{B_{\rho}\left(x_{0}\right)}\left|V_{p_{2}}\left(\frac{u-\xi-A\left(x-x_{0}\right)}{\rho}\right)\right|^{2} d x+\rho^{\alpha}\right] .
\end{aligned}
$$

The claim follows since $\rho^{p_{2} \alpha / 2} \leq \rho^{\alpha / 2}$.

\subsection{Approximate $\mathcal{A}$-harmonicity}

Lemma 9 Let $\bar{M}, \hat{M}>0$. Assume that $u$ is a local minimizer of the functional $\mathcal{F}$ with $\left(|D u|^{p_{2}}\right)_{x_{0}, 2 \rho} \leq \bar{M}$ and $A \in \operatorname{Hom}\left(\mathbb{R}^{n} ; \mathbb{R}^{N}\right)$ with $|A| \leq \hat{M}$. There exist a constant $c_{e} \equiv c_{e}\left(n, N, \gamma_{2}, L, \bar{M}, \hat{M}\right)$ and a radius $\rho_{0}=\rho_{0}(\alpha)$ such that for every ball $B_{\rho}\left(x_{0}\right) \Subset \Omega$ with $\rho \leq \rho_{0}$ we have

$$
\begin{aligned}
& \left|f_{B_{\rho}\left(x_{0}\right)} D^{2} f\left(x_{0}, A\right)(D u-A, D \varphi) d x\right| \\
& \leq c_{e}\left(\Phi^{2}+\sqrt{\nu_{\hat{M}, x_{0}}(\Phi)} \Phi+\sqrt{\omega(\rho)}\right) \sup _{B_{\rho}\left(x_{0}\right)}|D \varphi|,
\end{aligned}
$$

for all $\varphi \in C_{0}^{1}\left(B_{\rho}\left(x_{0}\right) ; \mathbb{R}^{N}\right)$.

Proof. First we assume $|D \varphi| \leq 1$. Let $0<s \leq 1$. We start by showing the following inequality:

$$
\begin{aligned}
& f_{B_{\rho}} D^{2} f\left(x_{0}, A\right)(D u-A, D \varphi) d x \\
& \geq \frac{1}{s} \int_{B_{\rho}} \int_{0}^{s}\left(D f\left(x_{0}, D u\right)-D f\left(x_{0}, D u+\tau D \varphi\right)\right) D \varphi d \tau d x \\
& \quad+f_{B_{\rho}} \int_{0}^{1}\left(D^{2} f\left(x_{0}, A\right)-D^{2} f\left(x_{0}, A+\tau(D u-A)\right)\right)(D u-A, D \varphi) d \tau d x \\
& \quad-\frac{1}{s} c \omega(\rho) \\
& =(A)+(B)+(C) .
\end{aligned}
$$

with $c \equiv c\left(\bar{M}, p_{2}, \delta\right)$. To see that let us start with the difference

$$
f_{B_{\rho}}\left(f\left(x_{0}, D u\right)-f\left(x_{0}, D u+s D \varphi\right)\right) d x .
$$


Introducing two additional differences we get

$$
\begin{gathered}
f_{B_{\rho}}\left(f\left(x_{0}, D u\right)-f\left(x_{0}, D u+s D \varphi\right)\right) d x=f_{B_{\rho}}\left(f\left(x_{0}, D u\right)-f(x, D u)\right) d x \\
+f_{B_{\rho}}(f(x, D u)-f(x, D u+s D \varphi)) d x \\
\quad+f_{B_{\rho}}\left(f(x, D u+s D \varphi)-f\left(x_{0}, D u+s D \varphi\right)\right) d x \\
\leq f_{B_{\rho}}\left|f(x, D u)-f\left(x_{0}, D u\right)\right| d x \\
\quad+f_{B_{\rho}}\left|f(x, D u+s D \varphi)-f\left(x_{0}, D u+s D \varphi\right)\right| d x \\
=(I)+(I I),
\end{gathered}
$$

since there holds

$$
f_{B_{\rho}}(f(x, D u)-f(x, D u+s D \varphi)) d x \leq 0,
$$

because of the minimality of the function $u$. To estimate the first term, we use the continuity condition for $f$ with respect to the variable $x$ as follows:

$$
\begin{gathered}
(I) \leq \int_{B_{\rho}} \omega\left(\left|x-x_{0}\right|\right)\left[\left(1+|D u|^{2}\right)^{p(x) / 2}+\left(1+|D u|^{2}\right)^{p\left(x_{0}\right) / 2}\right] . \\
\quad\left(1+\log \left(1+|D u|^{2}\right)\right) d x .
\end{gathered}
$$

By the elementary inequality

$$
\log \left(1+|z|^{2}\right) \leq C(a)|z|^{a} \quad \text { for all } 0<a<1,
$$

and the fact that $p_{2} \geq p(x)$ for all $x$ in $B_{2 \rho}\left(x_{0}\right)$ we see that

$$
\begin{aligned}
{\left[\left(1+|D u|^{2}\right)^{p(x) / 2}+\left(1+|D u|^{2}\right)^{p\left(x_{0}\right) / 2}\right]\left(1+\log \left(1+|D u|^{2}\right)\right) } & \\
& \leq c\left(p_{2}, \delta\right)\left(1+|D u|^{p_{2}(1+\delta / 4)}\right)
\end{aligned}
$$

where $\delta$ is the exponent of Lemma 2. Higher integrability of $u$ gives us (together with estimate (4.15) for the exponents)

$$
f_{B_{\rho}}|D u|^{p_{2}(1+\delta / 4)} d x \leq f_{B_{\rho}}\left(1+|D u|^{p(x)(1+\delta)}\right) d x \leq c\left(f_{B_{\rho}}\left(|D u|^{p(x)}+1\right) d x\right)^{1+\delta}
$$


This leads to

$$
(I) \leq c \omega(2 \rho)(1+\bar{M})^{1+\delta} .
$$

For $(I I)$ we follow the same way as for $(I)$, additionally using $|D \varphi| \leq 1$ : The continuity condition in the variable $x$ together with the estimates for the exponent $p$ lead to

$$
(I I) \leq \omega(2 \rho) f_{B_{\rho}}\left(1+|D u|^{2}+s^{2}|D \varphi|^{2}\right)^{p_{2} / 2}\left(1+\log \left(1+|D u|^{2}+s^{2}|D \varphi|^{2}\right)\right) d x .
$$

Using $|D \varphi| \leq 1$ we immediately get

$$
\begin{aligned}
(I I) & \leq c\left(p_{2}\right) \omega(2 \rho) f_{B_{\rho}}\left(1+|D u|^{p_{2}(1+\delta / 4)}\right) d x \\
& \leq c\left(\gamma_{2}, \delta\right) \omega(2 \rho)(1+\bar{M})^{1+\delta} .
\end{aligned}
$$

Finally we conclude

$$
f_{B_{\rho}}\left(f\left(x_{0}, D u\right)-f\left(x_{0}, D u+s D \varphi\right)\right) d x \leq c\left(\bar{M}, \gamma_{2}, \delta\right) \omega(2 \rho)
$$

and hence

$$
-f_{B_{\rho}} \int_{0}^{s} D f\left(x_{0}, D u+\tau D \varphi\right) D \varphi d \tau d x-c \omega(2 \rho) \leq 0 .
$$

Secondly we see that (remark that $\left.\varphi \in C_{0}^{1}\left(B_{\rho} ; \mathbb{R}^{N}\right)\right)$

$$
\begin{aligned}
f_{B_{\rho}} \int_{0}^{1} D^{2} f\left(x_{0}, A+\tau(D u-A)\right) & (D u-A, D \varphi) d \tau d x \\
& =f_{B_{\rho}}\left(D f\left(x_{0}, D u\right)-D f\left(x_{0}, A\right)\right) D \varphi d x \\
& =f_{B_{\rho}} D f\left(x_{0}, D u\right) D \varphi d x
\end{aligned}
$$

It follows then that (4.22) is true.

We start now taking a look at the right hand side of (4.22). In what follows we will distinguish the sets

$$
B_{\rho}^{+} \equiv B_{\rho} \cap\{x:|D u-A|>1\} \quad \text { and } \quad B_{\rho}^{-} \equiv B_{\rho} \cap\{x:|D u-A| \leq 1\} .
$$

Let us remark that by Lemma 5 in the case $|D u-A|>1$ we have for both $1<p_{2}<2$ and $p_{2} \geq 2$ the estimate

$$
|D u-A|^{p_{2}} \leq c\left(\gamma_{2}, \hat{M}\right)\left|V_{p_{2}}(D u-A)\right|^{2} \leq c\left|V_{p_{2}}(D u)-V_{p_{2}}(A)\right|^{2} .
$$


In the case $|D u-A| \leq 1$ we obtain for all $p_{2}>1$

$$
|D u-A|^{2} \leq c\left(\gamma_{2}, \hat{M}\right)\left|V_{p_{2}}(D u-A)\right|^{2} \leq c\left|V_{p_{2}}(D u)-V_{p_{2}}(A)\right|^{2} .
$$

We first estimate $|(A)|$. On the set $B_{\rho}^{-}$, we put $(A)$ in terms of the second derivative of $f$ by writing

$$
\begin{aligned}
\int_{0}^{s}\left(D f\left(x_{0}, D u\right)-\right. & \left.D f\left(x_{0}, D u+\tau D \varphi\right)\right) D \varphi d \tau \\
= & \int_{0}^{s} \int_{0}^{1} D^{2} f\left(x_{0}, D u+\sigma \tau D \varphi\right)(\tau D \varphi, D \varphi) d \sigma d \tau
\end{aligned}
$$

As we are on the set $B_{\rho}^{-}$, we have $|D u+\sigma \tau D \varphi| \leq|D u-A|+|A|+|D \varphi| \leq 2+\hat{M}$ and therefore $\left|D^{2} f\left(x_{0}, D u+\sigma \tau D \varphi\right)\right| \leq K(\hat{M})$, so we get

$$
\frac{1}{s}\left|\int_{0}^{s}\left(D f\left(x_{0}, D u\right)-D f\left(x_{0}, D u+\tau D \varphi\right)\right) D \varphi d \tau\right| \leq \frac{s}{2} K(\hat{M}) .
$$

On the set $B_{\rho}^{+}$by $(2.9)$ we get

$$
\begin{aligned}
& \frac{1}{s}\left|\int_{0}^{s}\left(D f\left(x_{0}, D u\right)-D f\left(x_{0}, D u+\tau D \varphi\right)\right) D \varphi d \tau\right| \\
& \quad \leq \frac{L}{s} \int_{0}^{s}\left[\left|D f\left(x_{0}, D u\right)\right|+\left|D f\left(x_{0}, D u+\tau D \varphi\right)\right|\right]|D \varphi| d \tau \\
& \quad \leq c\left(\gamma_{2}, L\right)\left[1+|D u|^{p_{2}-1}\right] .
\end{aligned}
$$

Since $|D u-A|>1$, we have $|D u|^{p_{2}-1} \leq|D u-A|^{p_{2}-1}+|A|^{p_{2}-1} \leq|D u-A|^{p_{2}}+$ $\hat{M}^{p_{2}-1} \leq\left(1+\hat{M}^{p_{2}-1}\right)|D u-A|^{p_{2}}$. Therefore the last term of the estimate above can be further estimated by

$$
c\left(\gamma_{2}, \hat{M}, L, n\right)|D u-A|^{p_{2}} \leq c\left|V_{p_{2}}(D u)-V_{p_{2}}(A)\right|^{2} .
$$

Summing up the arguments before we get

$$
|(A)| \leq c\left(n, L, \hat{M}, \gamma_{2}\right) f_{B_{\rho}}\left|V_{p_{2}}(D u)-V_{p_{2}}(A)\right|^{2} d x+\frac{s}{2} K(\hat{M})=c \Phi^{2}+\frac{s}{2} K(\hat{M}) .
$$

To estimate $|(B)|$, on the set $B_{\rho}^{-}$we use the fact that $|A+\tau(D u-A)| \leq \hat{M}+1$ and by (2.11) and (2.10) we obtain

$$
\begin{array}{rl}
\mid D^{2} & f\left(x_{0}, A\right)-D^{2} f\left(x_{0}, A+\tau(D u-A)\right) \mid \\
& =\left(\left|D^{2} f\left(x_{0}, A\right)-D^{2} f\left(x_{0}, A+\tau(D u-A)\right)\right|^{2}\right)^{1 / 2} \\
& \leq \sqrt{2 \sup _{|B| \leq \hat{M}+1}\left|D^{2} f(B)\right| \nu_{\hat{M}, x_{0}}(|D u-A|)} \\
& =\sqrt{2 K(\hat{M})} \sqrt{\nu_{\hat{M}, x_{0}}(|D u-A|)} .
\end{array}
$$


Therefore

$$
\begin{aligned}
\mid \int_{0}^{1} & {\left[D^{2} f\left(x_{0}, A\right)-D^{2} f\left(x_{0}, A+\tau(D u-A)\right)\right](D u-A, D \varphi) d \tau \mid } \\
& \leq \int_{0}^{1}\left|D^{2} f\left(x_{0}, A\right)-D^{2} f\left(x_{0}, A+\tau(D u-A)\right)\right||D u-A||D \varphi| d \tau \\
& \leq \sqrt{2 K(\hat{M})} \sqrt{\nu_{\hat{M}, x_{0}}(|D u-A|)}|D u-A| \\
& \leq c \sqrt{2 K(\hat{M})} \sqrt{\nu_{\hat{M}, x_{0}}\left(\left|V_{p_{2}}(D u)-V_{p_{2}}(A)\right|\right)}\left|V_{p_{2}}(D u)-V_{p_{2}}(A)\right| .
\end{aligned}
$$

On the set $B_{\rho}^{+}$we write

$$
\begin{aligned}
\mid \int_{0}^{1}\left(D^{2}\right. & \left.f\left(x_{0}, A\right)-D^{2} f\left(x_{0}, A+\tau(D u-A)\right)\right)(D u-A, D \varphi) d \tau \mid \\
= & \left|D^{2} f\left(x_{0}, A\right)(D u-A, D \varphi)+\left(D f\left(x_{0}, A\right)-D f\left(x_{0}, D u\right)\right) D \varphi\right| \\
= & :(*) .
\end{aligned}
$$

The first term is estimated by $K(\hat{M}+1)$. For the second term we use again the growth condition for $D f$ and follow exactly the same way as above for $|(A)|$ on the set $B_{\rho}^{+}$to get

$$
(*) \leq c|D u-A|^{p_{2}} \leq c\left|V_{p_{2}}(D u)-V_{p_{2}}(A)\right|^{2}
$$

with $c \equiv c\left(n, N, p_{2}, L, \bar{M}, \hat{M}\right)$. Putting the estimates together, we deduce

$$
|(B)| \leq c\left(\sqrt{\nu_{\hat{M}, x_{0}}(\Phi)} \Phi+\Phi^{2}\right) .
$$

We choose $s \equiv \sqrt{\omega(\rho)}<1$ and get

$$
|(A)|+|(B)|+|(C)| \leq c\left(\Phi^{2}+\sqrt{\nu_{\hat{M}, x_{0}}(\Phi)} \Phi+\sqrt{\omega(2 \rho)}\right)
$$

with a constant $c \equiv c\left(n, N, \gamma_{2}, L, \bar{M}, \hat{M}\right)$. Altogether we have shown

$$
f_{B_{\rho}} D^{2} f\left(x_{0}, A\right)(D u-A, D \varphi) d x \geq-c\left(\Phi^{2}+\sqrt{\nu_{\hat{M}, x_{0}}(\Phi)} \Phi+\sqrt{\omega(\rho)}\right) .
$$

The estimate for $f D f\left(x_{0}, A\right)(D u-A, D \varphi) d x$ from above is shown exactly in the same way. This gives the lemma for the case $|D \varphi| \leq 1$ and the general result can be achieved by rescaling. 


\section{Proof of the result}

\subsection{Excess-improvement lemma}

Let $M>0$ be fixed. Consider a point $x_{0} \in \Omega$ such that $\left|(D u)_{x_{0}, 2 \rho}\right| \leq M$ and $\Phi\left(x_{0}, 2 \rho\right) \leq 1$, where $\Phi$ is the function defined in (1.2) with $A=(D u)_{x_{0}, \rho}$, i.e.

$$
\Phi\left(x_{0}, \rho\right)=\Phi\left(x_{0}, \rho,(D u)_{x_{0}, \rho}\right)=\left(f_{B_{\rho}\left(x_{0}\right)}\left|V_{p_{2}}(D u)-V_{p_{2}}\left((D u)_{x_{0}, \rho}\right)\right|^{2} d x\right)^{1 / 2} .
$$

By properties of the function $V_{p_{2}}$ (Lemma 5$)$ we obtain

$$
\begin{aligned}
\left(|D u|^{p_{2}}\right)_{x_{0}, 2 \rho} \leq & 2^{p_{2}-1} \int_{B_{2 \rho}}\left|D u-(D u)_{x_{0}, 2 \rho}\right|^{p_{2}} d x+2^{p_{2}-1}\left|(D u)_{x_{0}, 2 \rho}\right|^{p_{2}} \\
\leq & 2^{p_{2}-1} c \int_{B_{2 \rho}}\left|V_{p_{2}}\left(D u-(D u)_{x_{0}, 2 \rho}\right)\right|^{2} d x \\
& +2^{p_{2}-1} c\left(f_{B_{2 \rho}} \mid V_{p_{2}}\left(D u-\left.(D u)_{x_{0}, 2 \rho}\right|^{2} d x\right)^{p_{2} / 2}+2^{p_{2}-1} M^{p_{2}}\right. \\
\leq & 2^{p_{2}-1} c_{1}(M)\left[\Phi^{p_{2}}\left(x_{0}, 2 \rho\right)+\Phi^{2}\left(x_{0}, 2 \rho\right)+M^{p_{2}}\right] \\
\leq & 2^{\gamma_{2}-1} c_{1}(M)\left[2+M^{\gamma_{2}}\right] \equiv \bar{M}
\end{aligned}
$$

where $c_{1}(M)$ is the constant out of Lemma 5. Furthermore we remark that by Hölder's inequality we immediately get

$$
\left|(D u)_{x_{0}, \rho}\right| \leq 2^{n}(|D u|)_{x_{0}, 2 \rho} \leq 2^{n}\left(|D u|^{p_{2}}\right)_{x_{0}, 2 \rho}^{1 / p_{2}} \leq 2^{n} \bar{M}^{1 / p_{2}} \equiv \hat{M} .
$$

By the quasiconvexity condition we get that $\mathcal{A} \equiv D^{2} f\left(x_{0},(D u)_{x_{0}, \rho}\right)$ is elliptic in the sense of Legendre-Hadamard with ellipticity constant $\kappa$ and upper bound $K$, where

$$
\kappa \equiv \frac{2}{L}\left(1+\hat{M}^{2}\right)^{\left(p_{2}-2\right) / 2} \quad \text { and } \quad K \equiv K(\hat{M}) \equiv \sup _{|A| \leq \hat{M}}\left|D^{2} f\left(x_{0}, A\right)\right| .
$$

Recall now the notation for constants. We will skip their dependence on $n, N, L$ and $\gamma_{1}, \gamma_{2}$ and remark their dependence on $M$ and $\alpha$. We will not remark the dependence on $\hat{M}$ and $\bar{M}$ since the two constants are computed out of $M, n, \gamma_{1}, \gamma_{2}$. Denote

$$
\begin{array}{ll}
c_{1}=c_{1}(M) & \text { from Lemma } 5(v) \text { and }(v i) \text { (properties of function } V), \\
c_{a}=c_{a}(M) & \text { from Lemma } 7 \text { (estimates for } \mathcal{A} \text {-harmonic function), } \\
c_{c}=c_{c}(M, \alpha) & \text { from Lemma } 8 \text { (Caccioppoli inequality), } \\
\rho_{0}=\rho_{0}(M, \alpha) & \text { from Lemma } 8 \text { (Caccioppoli inequality), } \\
c_{e}=c_{e}(M) & \text { from Lemma } 9 \text { (approximate } \mathcal{A} \text {-harmonicity). }
\end{array}
$$


Lemma 10 Let $M>0$ and $\beta \in(\alpha / 4,1)$ be fixed. There exist $\theta=\theta(\beta, M) \in$ $(0,1 / 4]$ and $\delta=\delta(\theta) \in(0,1]$ such that if $x_{0} \in \Omega$ is a point such that

$$
\begin{aligned}
\left|(D u)_{x_{0}, 2 \rho}\right| & \leq M, \\
\sqrt{\nu_{\hat{M}, x_{0}}\left(\Phi\left(x_{0}, \rho\right)\right)}+\Phi\left(x_{0}, \rho\right) & \leq \delta / 2, \\
2 \sqrt{2} c_{a} c_{1} c_{e} \sqrt{\Phi^{2}\left(x_{0}, \rho\right)+\frac{4}{\delta^{2}} \omega(\rho)} & \leq 1,
\end{aligned}
$$

hold for some $\rho \in\left(0, \rho_{0}\right]$, then

$$
\Phi^{2}\left(x_{0}, \theta \rho\right) \leq \theta^{2 \beta} \Phi^{2}\left(x_{0}, \rho\right)+\hat{c} \rho^{\alpha / 2},
$$

with $\hat{c} \equiv \hat{c}(M, \theta)$.

Proof. With $x_{0}$ fixed we will write $\Phi(\rho)$ instead of $\Phi\left(x_{0}, \rho\right)$. Let $\theta$ be a parameter, which is free at first and will be fixed at the end of the proof. Set

$$
\varepsilon \equiv \begin{cases}\theta^{n+4} & \text { if } p_{2} \in(1,2) \\ \theta^{n+p_{2}+2} & \text { if } p_{2} \geq 2\end{cases}
$$

and $\delta \equiv \delta(\varepsilon) \equiv \delta(\theta)$ be the parameter out of Lemma 6 (lemma on $\mathcal{A}$-harmonic approximation).

By Lemma $5(v)$ and $(v i)$ and using the fact that $\theta \leq 1$ we estimate

$$
\begin{aligned}
\Phi^{2}(\theta \rho) & =f_{B_{\theta \rho}\left(x_{0}\right)}\left|V_{p_{2}}(D u)-V_{p_{2}}\left((D u)_{x_{0}, \theta \rho}\right)\right|^{2} d x \\
\leq c & \int_{B_{\theta \rho}\left(x_{0}\right)}\left|V_{p_{2}}\left(D u-(D u)_{x_{0}, \rho}-\gamma D h\left(x_{0}\right)\right)\right|^{2} d x \\
& \quad+c\left|V_{p_{2}}\left((D u)_{x_{0}, \theta \rho}-(D u)_{x_{0}, \rho}-\gamma D h\left(x_{0}\right)\right)\right|^{2}
\end{aligned}
$$

with $c$ depending on $n, N, \gamma_{1}, \gamma_{2}$ and also on $M$ (the dependence on $M$ is due to the constant $\left.c_{1}(M)\right)$. We denote

$$
I \equiv f_{B_{\theta \rho}\left(x_{0}\right)}\left|V_{p_{2}}\left(D u-(D u)_{x_{0}, \rho}-\gamma D h\left(x_{0}\right)\right)\right|^{2} d x .
$$

To estimate the second expression on the right hand side of (5.27) we split the domain of integration into the subsets where $\left|D u-(D u)_{x_{0}, \rho}-\gamma D h\left(x_{0}\right)\right| \geq 1$ and $\left|D u-(D u)_{x_{0}, \rho}-\gamma D h\left(x_{0}\right)\right|<1$. Applying Lemma $5(i v)$ we obtain

$$
\begin{aligned}
\left|(D u)_{x_{0}, \theta \rho}-(D u)_{x_{0}, \rho}-\gamma D h\left(x_{0}\right)\right| & \leq \int_{B_{\theta \rho}\left(x_{0}\right)}\left|D u-(D u)_{x_{0}, \rho}-\gamma D h\left(x_{0}\right)\right| d x \\
& \leq \begin{cases}c\left(I^{1 / p_{2}}+I^{1 / 2}\right) & \text { for } p_{2} \in(1,2), \\
I^{1 / 2} & \text { for } p_{2} \geq 2 .\end{cases}
\end{aligned}
$$


Again applying Lemma $5(i v)$ we get

$$
\begin{aligned}
& V_{p_{2}}^{2}\left(I^{1 / p_{2}}+I^{1 / 2}\right) \leq c I \quad \text { for } p_{2} \in(1,2), \\
& V_{p_{2}}^{2}\left(I^{1 / 2}\right) \quad \leq c\left(I+I^{p_{2} / 2}\right) \text { for } p_{2} \geq 2 .
\end{aligned}
$$

Thus from (5.27) we conclude

$$
\Phi^{2}(\theta \rho) \leq \begin{cases}c I & \text { for } p_{2} \in(1,2), \\ c\left(I+I^{p_{2} / 2}\right) & \text { for } p_{2} \geq 2,\end{cases}
$$

with $c \equiv c(M)$. In this place we distinguish the cases $p_{2} \in(1,2)$ and $p_{2} \geq 2$. However, this is only for technical reasons, i.e. due to the difference in Lemma 5 (iv). We will see later that in fact $I$ is small and therefore we can skip the term $I^{p_{2} / 2}$ in the above estimate. We proceed then with the estimates for $I$.

Set

$$
\begin{aligned}
\gamma(\rho) & \equiv c_{1} c_{e} \sqrt{\Phi^{2}(\rho)+\frac{4}{\delta^{2}} \omega(\rho)}, \\
w & \equiv u-(D u)_{x_{0}, \rho}\left(x-x_{0}\right) .
\end{aligned}
$$

By Lemma $5(v i)$ and the smallness condition we have

$$
f_{B_{\rho}\left(x_{0}\right)}\left|V_{p_{2}}(D w)\right|^{2} d x \leq c_{1} \Phi^{2}(\rho) \leq \gamma^{2}
$$

It follows from Lemma 9 and the smallness condition that the function $w$ is approximate $\mathcal{A}$-harmonic, i.e.

$$
\begin{aligned}
\mid f_{B_{\rho}\left(x_{0}\right)} D^{2} f & \left(x_{0},(D u)_{x_{0}, \rho}\right)(D w, D \varphi) d x \mid \\
& \leq c_{e}\left(\Phi^{2}(\rho)+\sqrt{\nu_{\hat{M}, x_{0}}(\Phi(\rho))} \Phi(\rho)+\sqrt{\omega(\rho)}\right) \sup _{B_{\rho}\left(x_{0}\right)}|D \varphi| \\
& \leq c_{e}\left(\Phi(\rho) \frac{\delta}{2}+\sqrt{\omega(\rho)}\right) \sup _{B_{\rho}\left(x_{0}\right)}|D \varphi| \\
& \leq \gamma \delta \sup _{B_{\rho}\left(x_{0}\right)}|D \varphi| .
\end{aligned}
$$

Thus we are in the situation to apply Lemma 6 ( $\mathcal{A}$-harmonic approximation) providing a function $h \in W^{1, p_{2}}\left(B_{\rho}\left(x_{0}\right) ; \mathbb{R}^{N}\right)$ which is $D^{2} f\left(x_{0},(D u)_{x_{0}, \rho}\right)$-harmonic, such that

$$
f_{B_{\rho}\left(x_{0}\right)}\left|V_{p_{2}}(D h)\right|^{2} d x \leq c \quad \text { and } \quad f_{B_{\rho}\left(x_{0}\right)}\left|V_{p_{2}}\left(\frac{w-\gamma h}{\rho}\right)\right|^{2} d x \leq c \gamma^{2} \varepsilon
$$


Splitting the domain of integration in the first integral of (5.29) into the sets $\{|D h| \geq 1\}$ and $\{|D h|<1\}$ and using Lemma $5(i v)$ we obtain the upper bound for the mean value of $|D h|$ :

$$
f_{B_{\rho}\left(x_{0}\right)}|D h| d x \leq 2 \sqrt{2}
$$

Lemma 7 provides

$$
\rho \sup _{B_{\rho / 2}\left(x_{0}\right)}\left|D^{2} h\right|+\sup _{B_{\rho / 2}\left(x_{0}\right)}|D h| \leq c_{a} f_{B_{\rho}\left(x_{0}\right)}|D h| d x .
$$

By assumption there holds $(D u)_{x_{0}, \rho} \leq \hat{M}$ and by the smallness assumption $2 \sqrt{2} c_{a} \gamma \leq 1$, we conclude with

$$
\left|(D u)_{x_{0}, \rho}\right|+\gamma\left|D h\left(x_{0}\right)\right| \leq \hat{M}+\gamma c_{a} f_{B_{\rho}\left(x_{0}\right)}|D h| d x \leq \hat{M}+1 .
$$

Therefore we can apply the Caccioppoli inequality (Lemma 8) with $\xi \equiv \gamma h\left(x_{0}\right)$ and $A \equiv(D u)_{x_{0}, \rho}+\gamma D h\left(x_{0}\right)$ obtaining

$$
\begin{aligned}
I= & f_{B_{\theta \rho}\left(x_{0}\right)}\left|V_{p_{2}}\left(D u-(D u)_{x_{0}, \rho}-\gamma D h\left(x_{0}\right)\right)\right|^{2} d x \\
\leq & c_{c}\left[f_{B_{2 \theta \rho}\left(x_{0}\right)}\left|V_{p_{2}}\left(\frac{w-\gamma h\left(x_{0}\right)-\gamma D h\left(x_{0}\right)\left(x-x_{0}\right)}{2 \theta \rho}\right)\right|^{2} d x+\rho^{\alpha / 2}\right] \\
\leq & c\left[f_{B_{2 \theta \rho}\left(x_{0}\right)}\left|V_{p_{2}}\left(\frac{w-\gamma h}{2 \theta \rho}\right)\right|^{2} d x\right. \\
& \left.\quad+f_{B_{2 \theta \rho}\left(x_{0}\right)}\left|V_{p_{2}}\left(\gamma \frac{h-h\left(x_{0}\right)-D h\left(x_{0}\right)\left(x-x_{0}\right)}{2 \theta \rho}\right)\right|^{2} d x+\rho^{\alpha / 2}\right] .
\end{aligned}
$$

The first integral on the right hand side of (5.32) is estimated via (5.29) and Lemma $5(i)$

$$
\begin{aligned}
f_{B_{2 \theta \rho}\left(x_{0}\right)}\left|V_{p_{2}}\left(\frac{w-\gamma h}{2 \theta \rho}\right)\right|^{2} d x & \leq(2 \theta)^{-n} f_{B_{\rho}\left(x_{0}\right)}\left|V_{p_{2}}\left(\frac{w-\gamma h}{2 \theta \rho}\right)\right|^{2} d x \\
& \leq(2 \theta)^{-n} \max \left\{\left(\frac{1}{2 \theta}\right)^{2},\left(\frac{1}{2 \theta}\right)^{p_{2}}\right\} \gamma^{2} \varepsilon \\
& =c(n) \theta^{2} \gamma^{2},
\end{aligned}
$$

where the last inequality follows by the choice of $\varepsilon$ in (5.26). To estimate the second integral on the right hand side of (5.32) observe that by (5.30), (5.31), applying the Taylor theorem to $h$ on $B_{2 \theta \rho}\left(x_{0}\right)$ we obtain

$$
\sup _{B_{2 \theta \rho}\left(x_{0}\right)}\left|h(x)-h\left(x_{0}\right)-D h\left(x_{0}\right)\left(x-x_{0}\right)\right| \leq 4 \sqrt{2} c_{a} \theta^{2} \rho .
$$


Thus taking $\theta$ sufficiently small we have

$$
\left|\gamma \frac{h-h\left(x_{0}\right)-D h\left(x_{0}\right)\left(x-x_{0}\right)}{2 \theta \rho}\right| \leq 2 \sqrt{2} c_{a} \theta \gamma \leq 1 .
$$

Applying Lemma $5(i v)$ in both cases $p_{2} \in(1,2)$ and $p_{2} \geq 2$ we therefore obtain

$$
\begin{aligned}
f_{B_{2 \theta \rho}\left(x_{0}\right)} \mid V_{p_{2}} & \left.\left(\gamma \frac{h-h\left(x_{0}\right)-D h\left(x_{0}\right)\left(x-x_{0}\right)}{2 \theta \rho}\right)\right|^{2} d x \\
& \leq f_{B_{2 \theta \rho}\left(x_{0}\right)}\left|\gamma \frac{h-h\left(x_{0}\right)-D h\left(x_{0}\right)\left(x-x_{0}\right)}{2 \theta \rho}\right|^{2} d x \\
& \leq 8 c_{a}^{2} \theta^{2} \gamma^{2} .
\end{aligned}
$$

Alltogether, by (5.32), (5.33) and (5.34) we end up with

$$
I \leq c\left[c \theta^{2} \gamma^{2}+8 c_{a}^{2} \theta^{2} \gamma^{2}+\rho^{\alpha / 2}\right] .
$$

Taking $\theta$ and $\rho$ smaller if necessary we can have $I<1$. Therefore, since

$$
\gamma^{2} \equiv c_{1}^{2} c_{e}^{2}\left(\Phi^{2}(\rho)+\frac{4}{\delta^{2}} \rho^{\alpha}\right)
$$

it follows from (5.28) that

$$
\Phi^{2}(\theta \rho) \leq \tilde{c} \theta^{2} \Phi^{2}(\rho)+\hat{c} \rho^{\alpha / 2}
$$

with constants $\tilde{c} \equiv \tilde{c}(M, \alpha)$ and $\hat{c} \equiv \hat{c}(M, \theta, \delta, \alpha)$ (obviously the constants depend also on the structural constants $\left.n, N, L, \gamma_{1}, \gamma_{2}\right)$. We now fix $\theta$ sufficiently small, so that

$$
\tilde{c} \theta^{2} \leq \theta^{2 \beta}
$$

Choice of $\theta$ fixes $\epsilon$ and $\delta$ and the claim follows.

\subsection{Hölder continuity of $D u$ and regular set}

Let $M, \theta, \delta$ be fixed. If we assume that $\eta>0$ is such that

$$
\begin{aligned}
\sqrt{\nu_{\hat{M}, x_{o}}(\eta)}+\eta & \leq \delta / 2, \\
2 \sqrt{2} c_{1} c_{a} c_{e} \sqrt{\eta^{2}+\frac{4}{\delta^{2}} \omega(\rho)} & \leq 1
\end{aligned}
$$

and moreover it satisfies some additional technical smallness conditions, and $\rho_{1}$ is also sufficiently small, then, by a standard iteration technique we obtain

$$
\Phi^{2}\left(\theta^{j} \rho\right) \leq \theta^{2 \beta j} \Phi^{2}(\rho)+c(M, \alpha, \theta, \delta)\left(\theta^{j} \rho\right)^{\alpha / 2},
$$


for $j=1,2, \ldots$ provided

$$
\begin{aligned}
2 \rho & \leq \rho_{1}, \\
\left|(D u)_{x_{0}, 2 \rho}\right| & \leq \frac{M}{2}, \\
\Phi(\rho) & \leq \frac{\eta}{\sqrt{2}}, \\
\Phi(2 \rho) & \leq \frac{\eta}{\sqrt{2}} .
\end{aligned}
$$

We define

$$
\begin{aligned}
& \Sigma_{1} \equiv\left\{x_{0} \in \Omega: \limsup _{\rho \downarrow 0}\left|(D u)_{x_{0}, \rho}\right|<+\infty\right\}, \\
& \Sigma_{2} \equiv\left\{x_{0} \in \Omega: \underset{\rho \downarrow 0}{\left.\limsup f_{B_{\rho}\left(x_{0}\right)}\left|D u-(D u)_{x_{0}, \rho}\right| d x=0\right\} .}\right.
\end{aligned}
$$

The set $\Sigma_{1} \cap \Sigma_{2}$ is a set of full Lebesgue measure, i.e. $\mathcal{L}^{n}\left(\Omega \backslash\left(\Sigma_{1} \cap \Sigma_{2}\right)\right)=0$. The assumptions of Lemma 10 as well as the above conditions needed for iteration are satisfied in points $x_{0} \in \Sigma_{1} \cap \Sigma_{2}$. By standard interpolation we obtain an excessdecay estimate

$$
\Phi\left(x_{0}, r,(D u)_{r}\right) \leq c\left[\left(\frac{r}{\rho}\right)^{\beta} \Phi\left(x_{0}, \rho,(D u)_{\rho}\right)+\rho^{\alpha / 4}\right],
$$

for all $0 \leq r \leq \rho$ and with $\beta \in\left(\frac{\alpha}{4}, 1\right)$. The regularity result then follows from the fact that this excess-decay estimate implies

$$
\begin{aligned}
f_{B_{r}(x)}\left|V_{p_{2}}(D u)-\left(V_{p_{2}}(D u)\right)_{x, r}\right|^{2} d y & \leq f_{B_{r}(x)}\left|V_{p_{2}}(D u)-V_{p_{2}}\left((D u)_{x, r}\right)\right|^{2} d y \\
& \leq c\left[\left(\frac{r}{\rho}\right)^{\beta} \Phi\left(x_{0}, \rho,(D u)_{\rho}\right)+\rho^{\alpha / 4}\right]
\end{aligned}
$$

for any $x$ in the neighbourhood of $x_{0}$. From this estimate we conclude, by Campanato's characterization of Hölder continuous functions (see [6], [7]), that $V_{p_{2}}(D u)$ is Hölder continuous with the exponent $\frac{\alpha}{4}$ in a neighbourhood of $x_{0}$.

In order to pass over from Hölder continuity of the function $V_{p_{2}}(D u)$ to the function $D u$ itself, we use the following

Lemma 11 Let $p>1$ and $w: B \rightarrow \mathbb{R}^{N}$ a function such that the function $V_{p} \circ w:$ $B \rightarrow \mathbb{R}^{N}$ is Hölder continuous with an exponent $\alpha$. Then $w$ is Hölder continuous with the exponent $\beta:=\min \{1,2 / p\} \alpha$. 
From the above lemma we obtain that $D u$ is locally Hölder continuous with the exponent

$$
\min \left\{1,2 / \gamma_{2}\right\} \frac{\alpha}{4}
$$

\section{References}

[1] E. ACERBI and N. FUSCO, A regularity theorem for minimizers of quasiconvex integrals, Arch. Ration. Mech. Anal. 99 (1987), 261-281.

[2] E. ACERBI and G. MINGIONE, Regularity results for a class of functionals with nonstandard growth, Arch. Ration. Mech. Anal. 156(2) (2001), 121-140.

[3] E. ACERBI and G. MINGIONE, Regularity results for a class of quasiconvex functionals with nonstandard growth, Ann. Scuola Norm. Sup. Pisa Cl. Sci. IV 30(2) (2001), 311-339.

[4] E. ACERBI and G. MINGIONE, Regularity results for stationary electrorheological fluids, Arch. Ration. Mech. Anal. 164(3) (2002), 213-259.

[5] E. ACERBI and G. MINGIONE, Gradient estimates for the $p(x)$ - Laplacean system, J. Reine Angew. Math. 584 (2005), 117-148.

[6] S. CAMPANATO, Proprietà di una famiglia di spazi funzionali. Ann. Scuola Norm. Sup. Pisa 18(3) (1964), 137-160.

[7] S. CAMPANATO, Equazioni ellitichi del $I I^{e}$ ordine e spazi $\mathcal{L}^{2, \lambda}$. Ann. Mat. Pura Appl. 69 (1965), 321-382.

[8] M. CAROZZA, N. FUSCO and G. MingIONE. Partial regularity of minimizers of quasiconvex integrals with subquadratic growth. Ann. Mat. Pura Appl. 175(1) (1998), 141-164.

[9] F. DUZAAR, J. GROTOWSKI and M. KRONZ, Regularity of almost minimizers of quasi-convex variational integrals with subquadratic growth, Ann. Mat. Pura Appl. 184(4) (2005), 421-448.

[10] F. DUZAAR and K. STEFFEN, Optimal interior and boundary regularity for almost minimizers to elliptic variational integrals. J. Reine Angew. Math. 546 (2002), 73-138.

[11] I. EKELAND, Nonconvex minimization problems. Bull. Am. Math. Soc., New Ser. 1 (1979), 443-474.

[12] M. GIAQUINTA, Multiple Integrals in the Calculus of Variations and Nonlinear Elliptic Systems. Princeton University Press, 1983. 
[13] J. HABERMANN, Regularity results for functionals and Calderón-Zygmund estimates for systems of higher order with $p(x)$ growth. Ph.D Thesis, 2006.

[14] V. V. ZHIKOV, On Lavrentiev's phenomenon, Russian J. Math. Phys. 3 (1995), 249-269.

[15] V. V. ZHIKOV, On some variational problems, Russian J. Math. Phys. 5 (1997), 105-116.

Received 21 December 2006; accepted 13 March 2007

To access this journal online: http://www.birkhauser.ch 